\title{
Efecto de la Manipulación de la Temperatura Escrotal sobre las Características Clínicas, Seminales y Endocrinas en Carneros
}

\author{
Effect of Manipulation of Scrotal Temperature on Clinical, Seminal \\ and Endocrine Characteristics in RaMs
}

\author{
Wilfredo Huanca L. ${ }^{1}$, Luis Coronado S. ${ }^{2}$, David B. Galloway ${ }^{3}$
}

\section{Resumen}

\begin{abstract}
El objetivo del estudio fue evaluar los cambios en características clínicas, seminales y endocrinas en carneros con aislamiento escrotal térmico. Doce carneros Merino adultos fueron distribuidos al azar en un grupo Control $(n=4)$ y uno sometido a aislamiento escrotal $(n=8)$. A este grupo se le cubrió el escroto con una bolsa de algodón cubierta con plástico para elevar la temperatura a $39.0{ }^{\circ} \mathrm{C}$ por $48 \mathrm{~h}$. Se registró por cinco semanas el volumen del eyaculado, consistencia testicular, circunferencia escrotal, concentración espermática, motilidad masal e individual y tasa de anormalidades. El semen fue colectado tres veces por semana por electroeyaculación. La vena yugular fue canulada para colectar muestras por $6 \mathrm{~h}$ a intervalos de 10 min para la determinación de testosterona y LH plasmática mediante radioinmunoanálisis. Los resultados señalan cambios en circunferencia escrotal (de $30.43 \pm 1.1 \mathrm{~cm}$ al inicio a $25.00 \pm 0.5 \mathrm{~cm}$ en la semana 5); reducción de la concentración espermática (semana 2); motilidad masal: 0 (semana 2); motilidad individual: $2.45 \pm 3.1 \%$ (semana 2); y $82.3 \pm 29.3 \%$ de espermatozoides anormales (semana 3). A partir de la semana 2 disminuyen los niveles de testosterona $(280 \pm 83 \mathrm{pg} / \mathrm{ml})$ y se incrementan los de LH $(0.96 \pm 0.72 \mathrm{ng} / \mathrm{ml})$, incluyendo un mayor número de pulsos. Se concluye que el incremento de temperatura escrotal produce cambios en las características clínicas, seminales y endocrinas en el carnero.
\end{abstract}

Palabras clave: carnero, aislamiento escrotal, semen, hormona

\section{Abstract}

The aim of this study was to assess clinical and seminal characteristics and endocrine changes in rams with scrotal insulation. Twelve Merino rams were randomly distributed into a control group $(n=4)$ and a group with scrotal insulation $(n=8)$, that was done with a cotton bag coated with plastic to increase the temperature to $39.0{ }^{\circ} \mathrm{C}$ for $48 \mathrm{~h}$. The

\footnotetext{
${ }^{1}$ Laboratorio de Reproducción Animal, ${ }^{2}$ Laboratorio de Producción Agropecuaria, Facultad de Medicina Veterinaria, Universidad Nacional Mayor de San Marcos, Lima, Perú

${ }^{3}$ University of Melbourne, Australia

${ }^{4}$ E-mail:whuanca2002@yahoo.com
}

Recibido: 7 de diciembre de 2014

Aceptado para publicación: 10 de julio de 2015 
volume of ejaculate, testicular consistency, scrotal circumference, sperm concentration, mass and individual motility, and sperm abnormalities were recorded for five weeks. Semen collection by electroejaculation was conducted three times per week. The animals were cannulated in the jugular vein and blood samples were taken for 6 hours at 10 minute intervals for testosterone and LH determination by radioimmunoassay. The results showed changes in scrotal circumference (from $30.43 \pm 1.1$ to $25.00 \pm 0.5 \mathrm{~cm}$ on week 5); reduction of sperm concentration (week 2); mass motility: 0 (week 2); individual motility: $2.45 \pm 3.1$ (week 2); and $82.3 \pm 29.3 \%$ sperm abnormalities at week 3 . Testosterone concentration decreased $(280 \pm 83 \mathrm{pg} / \mathrm{ml})$ and LH concentrations increased $(0.96 \pm 0.72 \mathrm{ng} / \mathrm{ml})$ at week 2 , including higher number of LH pulses. It is concluded that the increase of scrotal temperature causes changes in the clinical, seminal, and in the secretion of testosterone and LH.

Key words: rams, scrotal insulation, semen, hormone

\section{INTRODUCCIÓN}

El óptimo comportamiento reproductivo de los mamíferos está influenciado por factores nutricionales, genéticos, sanitarios y medioambientales. Los tres primeros pueden ser medianamente controlados; sin embargo, el medio ambiente puede ser difícil de acondicionar, y el hombre solo se puede limitar a diseñar alternativas para mejorar las condiciones de alojamiento de los animales.

En el caso de mamíferos, la fisiología en el macho ha permitido que los testículos, órganos primarios de la reproducción, puedan ser regulados acorde a los cambios climáticos. Para su óptimo funcionamiento, el testículo requiere mantenerse a una temperatura de 4 a 5 grados menor que la corporal, donde el escroto y el sistema vascular a nivel testicular actúan como elementos termorreguladores, mediante un mecanismo de intercambio de calor a nivel del cordón espermático. La arteria testicular se alarga conforme el testículo se desplaza al escroto, y la elongación es mayor que la necesaria para poder seguir los movimientos del testículo durante la regulación térmica (Setchell et al., 1994).
El estrés térmico escrotal produce alteraciones en la función testicular, pérdida del peso testicular, pérdida de células germinales, incremento en la apoptosis celular (Lue et al., 1999), disminución de la concentración, viabilidad y motilidad espermática (Zhu et al., 2004; Banks et al., 2005), y una reducción de la capacidad fecundante de los espermatozoides causando infertilidad temporal (Setchell, 1998; Yaeram et al., 2006).

El efecto del estrés térmico sobre las células de la línea germinal masculina depende del grado de apoptosis o necrosis y de la capacidad de estas células de responder al estrés y reparar su ADN (Rockett et al., 2001). Las células de cada una de las fases de la espermatogénesis tienen diferentes respuestas al estrés térmico. Algunas células se eliminan mediante apoptosis, mientras que otras células germinales completan su desarrollo y dan lugar a espermatozoides motiles con daño en su ADN (Banks et al., 2005). Asimismo, los espermatocitos son las células más vulnerables al estrés térmico (Banks et al., 2005; Paul et al., 2008).

En el ratón, se han reportado alteraciones en la síntesis de ADN, ARN y proteínas, así como un empaquetamiento anormal de la 
cromatina y una reducción de la integridad del ADN espermático (Banks et al., 2005), como consecuencia del incremento de la temperatura escrotal. A nivel molecular, se describe una reducción en la expresión génica, particularmente de genes implicados en la reparación del $\mathrm{ADN}$ y la recombinación, la regulación del ciclo celular y la respuesta al estrés (Rockett et al., 2001).

El incremento de la temperatura a nivel escrotal llega a causar una reducción del 40 a $60 \%$ del tamaño del testículo, observándose los principales cambios durante la segunda semana del tratamiento térmico (Byers y Glover, 1984; Sanford y Baker, 1990). La reducción del testículo es atribuida a una disminución significativa del diámetro del túbulo seminífero y a una disminución del volumen total del tejido intersticial (Hansen, 2009). Asimismo, se observan cambios en la concentración espermática, a partir de los 14-16 días (Setchell, 1987; McLaren, 1998), aunque el volumen no se altera (Karagiannidis et al., 2000). Igualmente, se ha reportado una alta incidencia de anormalidades en cabeza $(50 \%)$ y cola $(80 \%)$ por efecto del calor (Williamson, 1974).

Existen pocos estudios referentes a cambios ocasionados por el estrés térmico sobre la secreción de hormonas reproductivas. En toros y verracos, el calor origina una disminución de las concentraciones circulantes de testosterona pero estos niveles vuelven a los niveles previos si continúa el estrés térmico (Hansen, 2009). La disminución de testosterona a nivel sanguíneo puede explicarse por una disminución en el número de receptores a nivel de las células de Leydig y un incremento en los niveles sanguíneos de LH y FSH (Byers y Glover, 1984). En hembras, el estrés térmico altera la secreción de LH, aún en ausencia de ovarios, $\mathrm{y}$ se sugiere que puede presentar una conducta similar en el macho (Hansen, 2009).
El presente estudio está orientado a determinar las variaciones en las características clínicas, seminales y endocrinas a nivel testicular, en carneros sometidos a efecto térmico a nivel testicular.

\section{Materiales y Métodos}

\section{Lugar del Estudio y Animales}

El experimento fue realizado en el Centro Clínico Veterinario de la Facultad de Ciencias Veterinarias, Universidad de Melbourne, Australia, ubicado en Werribee, a $35 \mathrm{~km}$ de la ciudad de Melbourne. La fase experimental se desarrolló en los meses de verano, con una temperatura ambiental media de $35.3 \pm$ $3.8^{\circ} \mathrm{C}$.

Se utilizaron 12 carneros de raza Merino, con una edad promedio de 18 meses, sexualmente maduros y en buena condición corporal. Los animales fueron mantenidos bajo las mismas condiciones de manejo y alimentación, siendo colocados en jaulas colectivas y con alimentación a base de heno de alfalfa y agua a discreción.

\section{Diseño Experimental}

a) Fase pre-experimental: Correspondiente a la semana previa del experimento. Se realizaron evaluaciones clínicas testiculares y de las características seminales.

b) Fase experimental: Periodo de 35 días, donde el primer día se colocaron las bolsas escrotales por 48 horas a 8 carneros (grupo tratado), quedando los 4 restantes sin aislamiento escrotal (grupo control).

Para el aislamiento escrotal, los testículos fueron cubiertos con una bolsa de algodón recubierta con bolsas de plástico para obtener una temperatura escrotal de $39 \pm 0.5{ }^{\circ} \mathrm{C}$. 


\section{Evaluaciones}

\section{Consistencia testicular}

La consistencia testicular se evaluó acorde con el método de McGowan et al. (1995). Se hicieron tres evaluaciones preexperimentales y cada tres días durante la fase experimental, conjuntamente con la colección de semen. Se consideraron valores de 1 a 5 , siendo 1 de muy baja y 5 de muy firme y muy alta elasticidad. Los valores de firmeza y elasticidad de 2:2, 2:3 y 3:3 fueron considerados normales. Adicionalmente, se midió la circunferencia escrotal, basado en la técnica descrita por Chenoweth y Kastelic (2007).

\section{Colección y evaluación de semen}

Se hicieron tres colecciones de semen por semana mediante electroeyaculación (Bailey de 6 voltios), aplicando un estímulo eléctrico vía rectal por 6 segundos, seguido de un periodo de descanso de 2 segundos, con reinicio de los ciclos de estímulo en orden creciente hasta conseguir la erección y eyaculación. El semen colectado fue mantenido a $35^{\circ} \mathrm{C}$.

En la evaluación del semen se siguió la técnica descrita por Evans y Maxell (1987), donde se determinó el volumen $(\mathrm{ml})$, color, motilidad masal (0 a 5), motilidad individual (0 a 100\%), concentración, y porcentajes de espermatozoides vivos y muertos y anormales (cola y cabeza), mediante conteos mínimos de 200 espermatozoides por campo.

\section{Determinación de LH y testosterona}

Se colectaron muestras de sangre los días $-1,7,14$ y 28 de la fase experimental. Para esto, se canuló la vena yugular el día previo y los animales fueron colocados en jaulas individuales. El muestreo se realizó por un periodo de 6 horas, a intervalos de 10 minutos, obteniéndose un total de 36 muestras por animal por día de colección.

La muestra de sangre $(10 \mathrm{ml})$ fue colectada en tubos con heparina al $0.9 \%$ y fue centrifugada dentro de los 10 minutos de su colección. El plasma resultante fue almacenado en congelación a $-20{ }^{\circ} \mathrm{C}$. Para el análisis de LH se utilizaron todas las muestras $(\mathrm{n}=36)$ y para el análisis de testosterona se utilizó un pool de las muestras tomadas en cada muestreo por animal. Las concentraciones de LH y testosterona fueron determinadas mediante la técnica de radioinmunoanálisis utilizándose un kit comercial de DPC (Diagnostic Products Corporation, CA, EEUU) de fase sólida para la determinación de testosterona y doble anticuerpo para la determinación de LH. Los niveles de testosterona fueron expresados en $\mathrm{pg} / \mathrm{ml}$ y los de LH en $\mathrm{ng} / \mathrm{ml}$.

\section{Análisis Estadístico}

Se utilizó la prueba T student para comparar diferencias entre los tratamientos en cada semana posterior al aislamiento para las variables en estudio.

\section{Resultados}

\section{Evaluación Clínica Testicular}

La circunferencia escrotal fue de 30.43 $\mathrm{cm}$ al inicio del estudio, mostrando una reducción significativa en la segunda semana $(28.19 \mathrm{~cm} ; \mathrm{p}<0.01)$, llegando a medir $25.0 \mathrm{~cm}$ en la quinta semana, mientras que las medidas de circunferencia escrotal en el grupo control no registraron cambios significativos (Cuadro 1). Por otro lado, no se observaron valores anormales en consistencia testicular Cuadro 1). 
Cuadro 1. Circunferencia escrotal y consistencia testicular en carneros sometidos a estrés térmico escrotal $\left(39^{\circ} \mathrm{C}\right)$ por 48 horas

\begin{tabular}{ccccc}
\hline \multirow{2}{*}{$\begin{array}{c}\text { Periodo } \\
\text { (semanas })\end{array}$} & $\begin{array}{c}\text { Control } \\
\text { Circunferencia } \\
(\mathrm{cm} \pm \text { d.e. })\end{array}$ & $\begin{array}{c}\text { Consistencia } \\
(1 \mathrm{a} 5)\end{array}$ & $\begin{array}{c}\text { Circunferencia } \\
(\mathrm{cm} \pm \text { d.e. })\end{array}$ & $\begin{array}{c}\text { Consistencia } \\
(1 \mathrm{a} 5)\end{array}$ \\
\hline Inicio & $29.81 \pm 1.3^{\mathrm{a}}$ & 2.0 & $30.43 \pm 1.1^{\mathrm{a}}$ & 2.0 \\
1 & $30.25 \pm 1.2^{\mathrm{a}}$ & 2.0 & $29.61 \pm 1.8^{\mathrm{a}}$ & 2.0 \\
2 & $30.55 \pm 1.3^{\mathrm{a}}$ & 3.0 & $28.19 \pm 1.2^{\mathrm{b}}$ & 3.0 \\
3 & $30.16 \pm 1.2^{\mathrm{a}}$ & 3.0 & $26.10 \pm 1.2^{\mathrm{b}}$ & 2.0 \\
4 & $29.42 \pm 0.6^{\mathrm{a}}$ & 2.0 & $25.58 \pm 0.6^{\mathrm{b}}$ & 3.0 \\
5 & $30.42 \pm 0.4^{\mathrm{a}}$ & 3.0 & $25.00 \pm 0.5^{\mathrm{b}}$ & 2.0 \\
\hline
\end{tabular}

${ }^{a, b}$ Superíndices diferentes dentro de filas indican diferencia estadística $(p \varangle 0.01)$

Cuadro 2. Volumen de semen colectado y concentración espermática en carneros some tidos a estrés térmico escrotal $\left(39^{\circ} \mathrm{C}\right)$ por 48 horas

\begin{tabular}{ccccc}
\hline \multirow{2}{*}{ Semanas } & \multicolumn{2}{c}{$\begin{array}{c}\text { Volumen } \\
(\mathrm{ml} \pm \text { d.e. })\end{array}$} & \multicolumn{2}{c}{$\begin{array}{c}\text { Concentración espermática } \\
(\mathrm{x} \mathrm{10} / \mathrm{ml} \pm \text { d.e. })\end{array}$} \\
\cline { 2 - 5 } & Control & $\begin{array}{c}\text { Aislamiento } \\
\text { escrotal }\end{array}$ & Control & $\begin{array}{c}\text { Aislamiento } \\
\text { esc rotal }\end{array}$ \\
\hline Inicio (0) & $0.70 \pm 0.29$ & $0.73 \pm 0.32$ & $2.88 \pm 0.37^{\mathrm{a}}$ & $3.08 \pm 0.43^{\mathrm{a}}$ \\
1 & $0.71 \pm 0.33$ & $0.75 \pm 0.21$ & $3.02 \pm 0.60^{\mathrm{a}}$ & $2.81 \pm 0.41^{\mathrm{a}}$ \\
2 & $0.97 \pm 0.08$ & $0.87 \pm 0.18$ & $2.96 \pm 0.42^{\mathrm{a}}$ & $1.92 \pm 0.34^{\mathrm{b}}$ \\
3 & $0.96 \pm 0.10$ & $0.92 \pm 0.10$ & $3.10 \pm 0.19^{\mathrm{a}}$ & $1.34 \pm 0.20^{\mathrm{b}}$ \\
4 & $0.86 \pm 0.22$ & $0.86 \pm 0.21$ & $3.17 \pm 0.30^{\mathrm{a}}$ & $1.41 \pm 0.23^{\mathrm{b}}$ \\
5 & $0.93 \pm 0.10$ & $0.91 \pm 0.22$ & $3.06 \pm 0.23^{\mathrm{a}}$ & $1.04 \pm 0.19^{\mathrm{b}}$ \\
\hline
\end{tabular}

${ }^{a, b}$ Superíndices diferentes dentro de filas en la concentración espermática indican diferencia estadística $(p<0.01)$

\section{Volumen Seminal y Concentración Espermática}

No se determinaron diferencias significativas en volumen seminal, donde los valores variaron de 0.73 a $0.92 \mathrm{ml}$ en los animales bajo aislamiento escrotal y de 0.70 a 0.97 en los animales del grupo control (Cuadro 2).
La concentración espermática inicial fue $3.08 \times 10^{6} / \mathrm{ml}$, disminuyendo a $2.81 \times 10^{6} / \mathrm{ml}$ en la segunda semana, llegando a $1.04 \times 10^{6}$ $\mathrm{ml}$ en la quinta semana, habiendo diferencias significativas en relación al grupo control $(\mathrm{p}<0.01)$ (Cuadro 2). 
Cuadro 3. Motilidad masal (0 a 5) y motilidad progresiva individual (\%) en carneros sometidos a estrés térmico escrotal $\left(39^{\circ} \mathrm{C}\right)$ por 48 horas

\begin{tabular}{ccccc}
\hline \multirow{2}{*}{ Semana } & \multicolumn{2}{c}{$\begin{array}{c}\text { Motilidad masal } \\
(0-5)\end{array}$} & \multicolumn{2}{c}{$\begin{array}{c}\text { Motilidad individual } \\
(\%)\end{array}$} \\
\cline { 2 - 5 } & Control & $\begin{array}{c}\text { Aislamiento } \\
\text { escrotal }\end{array}$ & Control & $\begin{array}{c}\text { Aislamiento } \\
\text { escrotal }\end{array}$ \\
\cline { 2 - 5 } & $5.6 \pm 0.4^{\mathrm{a}}$ & $5.5 \pm 0.4^{\mathrm{a}}$ & $88.7 \pm 4.5^{\mathrm{a}}$ & $85.5 \pm 5.2^{\mathrm{a}}$ \\
\hline Inicio (0) & $5.8 \pm 0.5^{\mathrm{a}}$ & $3.8 \pm 0.4^{\mathrm{a}}$ & $85.4 \pm 5.3^{\mathrm{a}}$ & $56.3 \pm 4.3^{\mathrm{b}}$ \\
1 & $5.7 \pm 0.3^{\mathrm{a}}$ & $0^{\mathrm{b}}$ & $85.8 \pm 4.4^{\mathrm{a}}$ & $2.5 \pm 3.1^{\mathrm{b}}$ \\
2 & $5.5 \pm 0.4^{\mathrm{a}}$ & $0^{\mathrm{b}}$ & $85.6 \pm 4.5^{\mathrm{a}}$ & $0^{\mathrm{b}}$ \\
3 & $5.8 \pm 0.3^{\mathrm{a}}$ & $0^{\mathrm{b}}$ & $73.5 \pm 5.2^{\mathrm{a}}$ & $0^{\mathrm{b}}$ \\
4 & $5.4 \pm 0.5^{\mathrm{a}}$ & $0.3 \pm 0.5^{\mathrm{b}}$ & $83.5 \pm 4.2^{\mathrm{a}}$ & $1.2 \pm 2.7^{\mathrm{b}}$ \\
\hline 5
\end{tabular}

${ }^{a, b}$ Superíndices diferentes dentro de filas y variable indican diferencia estadística $(p \varangle 0.01)$

\section{Motilidad Masal e Individual Progresiva}

Se observan diferencias en motilidad masal entre los dos grupos a partir de la primera semana (5.8 y 3.8 , respectivamente), llegando a niveles de 0 a partir de la segunda semana en el grupo con aislamiento escrotal (Cuadro 3). En lo referente a la motilidad individual progresiva, los cambios ocurren en forma similar a partir de la primera semana, donde la motilidad desciende a $56.3 \%$, llegando a niveles imperceptibles a partir de la segunda semana (Cuadro 3).

\section{Morfología Espermática}

El porcentaje de espermatozoides anormales en el grupo tratado se incrementa a partir de la segunda semana (22.8\%) llegando al $99 \%$ a partir de la tercera semana (Cuadro 4; $<<0.01)$. La principal anormalidad presente fue de cabeza sola $(2.7$ a $3.5 \%$ en el grupo control vs. 14.3 a $66.8 \%$ en el grupo con aislamiento escrotal) y cola doblada $(0.25$ a $0.75 \%$ en el grupo control vs 0.8 a $13.3 \%$ en el grupo con aislamiento escrotal).
Cuadro 4. Presencia de espermatozoides anormales $(\% \pm$ de) en carneros sometidos a estrés térmico escrotal $\left(39^{\circ} \mathrm{C}\right)$ por 48 horas

\begin{tabular}{ccc}
\hline Semanas & Control & $\begin{array}{c}\text { Aislamiento } \\
\text { escrotal }\end{array}$ \\
\hline Inicio (0) & $8.3 \pm 4.8^{\mathrm{a}}$ & $10.8 \pm 4.4^{\mathrm{a}}$ \\
1 & $7.5 \pm 5.4^{\mathrm{a}}$ & $22.8 \pm 18.4^{\mathrm{b}}$ \\
2 & $6.5 \pm 8.3^{\mathrm{a}}$ & $82.3 \pm 29.3^{\mathrm{b}}$ \\
3 & $9.8 \pm 6.7^{\mathrm{a}}$ & $99.7 \pm 0.0^{\mathrm{b}}$ \\
4 & $10.3 \pm 8.2^{\mathrm{a}}$ & $99.1 \pm 0.1^{\mathrm{b}}$ \\
5 & $21.0 \pm 7.3^{\mathrm{a}}$ & $99.1 \pm 0.1^{\mathrm{b}}$ \\
\hline a,b & Superíndices diferentes \\
filas indican diferencia de \\
(p<0.01)
\end{tabular}

\section{Niveles Hormonales}

Los niveles de testosterona disminuyen en el grupo con aislamiento escrotal a partir de la segunda semana $(280 \mathrm{pg} / \mathrm{ml})$ y se mantuvieron en esos niveles hasta el final del es- 
Cuadro 6. Concentración (promedio \pm de) de hormona luteinizante (LH) plasmática $(\mathrm{ng} / \mathrm{ml})$ en carneros sometidos a estrés térmico escrotal $\left(39^{\circ} \mathrm{C}\right)$ por 48 horas

\begin{tabular}{ccccc}
\hline & \multicolumn{2}{c}{ Control } & \multicolumn{2}{c}{ Aislamiento esc rotal } \\
\cline { 2 - 5 } Muestra & $(\mathrm{ng} / \mathrm{ml})$ & $\begin{array}{c}\text { Pulsos } \\
(\text { en 6 horas })\end{array}$ & $(\mathrm{ng} / \mathrm{ml})$ & $\begin{array}{c}\text { Pulsos } \\
(\text { en 6 horas })\end{array}$ \\
\hline Día 0 & $0.07 \pm 0.06$ & $0.04 \pm 0.40$ & $0.07 \pm 0.06$ & $0.04 \pm 0.40$ \\
Día 7 & $0.08 \pm 0.07$ & $0.05 \pm 0.40$ & $0.96 \pm 0.72$ & $0.92 \pm 0.33$ \\
Día 14 & $0.07 \pm 0.07$ & $0.04 \pm 0.40$ & $2.29 \pm 0.88$ & $1.20 \pm 0.37$ \\
Día 28 & $0.08 \pm 0.06$ & $0.04 \pm 0.40$ & $2.11 \pm 0.92$ & $1.32 \pm 0.43$ \\
\hline
\end{tabular}

Cuadro 5. Concentración (promedio \pm de) de testosterona plasmática $(\mathrm{pg} / \mathrm{ml})$ en carneros sometidos a estrés térmico escrotal $\left(39^{\circ} \mathrm{C}\right)$ por 48 horas

\begin{tabular}{lcc}
\hline Muestra & Control & $\begin{array}{c}\text { Aislamiento } \\
\text { escrotal }\end{array}$ \\
\hline Día 0 & $436 \pm 202^{\mathrm{a}}$ & $483 \pm 237^{\mathrm{a}}$ \\
Día 7 & $487 \pm 219^{\mathrm{a}}$ & $280 \pm 83^{\mathrm{b}}$ \\
Día 14 & $408 \pm 201^{\mathrm{a}}$ & $231 \pm 67^{\mathrm{b}}$ \\
Día 28 & $459 \pm 238^{\mathrm{a}}$ & $201 \pm 63^{\mathrm{b}}$ \\
\hline a,b & Superíndices diferentes & dentro de \\
filas indican diferencia & estadística \\
( $p<0.01)$ &
\end{tabular}

tudio (Cuadro 5). En cambio, se observa una tendencia opuesta en los perfiles de LH, con un incremento en la concentración desde 0.07 $\mathrm{ng} / \mathrm{ml}$ al inicio del estudio hasta $2.29 \mathrm{ng} / \mathrm{ml}$ en el tercer muestreo y con un incremento en el número de pulsos de $0.04 \mathrm{al}$ inicio del estudio hasta 1.32 pulsos/6 horas en el día 28 (Cuadro 6).

\section{Discusión}

La reducción de la circunferencia escrotal en animales sometidos al aislamiento escrotal observado en el presente estudio es coincidente con resultados de otros estudios (Hochereau-de Reviers et al., 1993; Setchell, 1998). Esta reducción de la circunferencia escrotal se explica porque la acción del calor origina alteraciones a nivel de los túbulos seminíferos, con una reducción de la luz del túbulo pero no en su longitud (Hochereau-de Reviers et al., 1993). Estas alteraciones del tejido testicular se deberían reflejar en una alteración de la consistencia testicular; sin embargo, los resultados observados en el presente experimento no coinciden con lo esperado. Esto podría atribuirse a que si bien existe reducción de la luz de los túbulos, estos aun contienen espermatozoides y, además, no se ha producido una reducción del tejido intersticial, según ha sido señalado por Hochereau-de Reviers et al. (1993).

Si bien no hubo diferencias significativas en el volumen seminal entre los dos grupos en las cinco semanas del estudio, la con- 
centración espermática se vio afectada a partir de la segunda semana (Cuadro 2). La concentración esperada de espermatozoides en carneros varía entre 1.5 y 3.0 millones $/ \mathrm{ml}$; sin embargo, en el presente estudio se llegaron a observar valores de 1.04 millones $/ \mathrm{ml}$. Los espermatocitos primarios son las primeras células en ser afectadas por el estrés térmico, en tanto que el efecto del aislamiento escrotal es menos marcado a nivel del epidídimo (Setchell et al., 1994), lugar de almacenamiento de los espermatozoides producidos, de allí que el efecto del aislamiento escrotal no se observó durante la primera semana del estudio.

El efecto del incremento de la temperatura sobre una reducción de la motilidad masal se debe a una menor concentración de espermatozoides y al incremento de formas anormales y espermatozoides muertos en el eyaculado. Asimismo, las proteínas específicas dineina y tubulina, necesarias para estimular la motilidad espermática, mediante una acción enzimática sobre el ATP, para producir energía necesaria para el movimiento del axonema, se encuentran afectadas por el aumento de la temperatura escrotal (Huang et al., 2005).

Las anormalidades más frecuentes en los espermatozoides fueron las de cabezas solas y colas dobladas, las cuales pueden clasificarse como anormalidades primarias, debido a que la alteración se produce a nivel del proceso espermatogénico (Sorensen, 1991). Las células afectadas por el estrés térmico escrotal son las células germinales presentes en el testículo y que participan en la espermatogénesis y los espermatozoides del epidídimo (Banks et al., 2005; Zhu et al., 2004). La intensidad del efecto del estrés térmico sobre las células de la línea germinal es dependiente de la capacidad de estas células en responder al estrés y reparar el ADN y la posibilidad de sufrir apoptosis o necrosis (Rockett et al., 2001).
El incremento del porcentaje de espermatozoides a partir de la segunda semana posterior al aislamiento escrotal se explica porque ya se han liberado los espermatozoides almacenados en el epidídimo y están siendo reemplazados por espermatozoides que se han desarrollado durante el proceso de estrés térmico (Sorensen, 1991).

El incremento de la temperatura testicular produce una reducción del tejido intersticial, afectando el número de células de Leydig (Byers y Glovers, 1984). Es así que una reducción de estas células afecta la producción de testosterona, lo cual se vio reflejado en la disminución de los niveles plasmáticos de testosterona a partir de la segunda semana del aislamiento escrotal (280 $\pm 83 \mathrm{pg} / \mathrm{ml}$ ). Por otro lado, el comportamiento opuesto en la secreción de LH se debe al descenso de testosterona y, por lo tanto, a la ausencia del mecanismo de retroalimentación negativa sobre el eje hipotalámico-hipofisario, de modo que permite una secreción continua de LH, lo cual fue demostrado por Byers y Glover (1984).

\section{Conclusiones}

El incremento de la temperatura testicular con bolsas térmicas en carneros produce cambios en las características clínicas, seminales y endocrinas. Es así que ocasiona una disminución de la circunferencia escrotal, disminución en la concentración de espermatozoides, motilidad masal y motilidad individual, y en los niveles circulantes de testosterona. Por otro lado, incrementa el porcentaje de espermatozoides anormales y muertos, así como un incremento en la secreción de LH, tanto en cantidad como en la frecuencia pulsátil.

\section{Literatura Citada}

1. Banks S, King SA, Irvine DS, Saunders PT. 2005. Impact of a mild- 
scrotal heat stress on DNA integrity in murine spermatozoa. Reproduction 129: 505-514. doi: 10.1530/rep.1.00531

2. Byers SW, Glover TD. 1984. Effect of scrotal insulation on the pituitary Testicular axis of the ram. J Reprod Fert 71:23-31. doi: 10.1530/jrf.0.0710023

3. Chenoweth PT, Kastelic JP. 2007. Clinical reproductive physiology and endocrinology of bulls. In: Youngquist, RS; Threlfall, WR (eds). Current theraphy in large animal theriogenology 2. USA: Saunders. p 221-228.

4. Evans G, Maxwell WMC. 1987. Salamon's artificial insemination of sheep and goats. Sydney: Butterworths. $185 \mathrm{p}$.

5. Hansen P. 2009. Effects of heat stress on mammalian reproduction. Philos Trans R Soc Lond B Biol Sci 364: 33413350. doi: 10.1098/rstb.2009.0131

6. Hochereau-de Reviers MT, Locatelli $A$, Perreau C, Pisselet C, Setchell BP. 1993. Effect of a single brief period of moderate heating of the testes on seminiferous tubules in hypophysectomized rams treated with pituitary extract. J Reprod Fert 97: 381-387. doi: 10.1530/jrf.0.0970381

7. Huang CC, Lin DP, Tsao HM, Cheng TC, Liu CH, Lee MS. 2005. Sperm DNA fragmentation negatively correlates with velocity and fertilization rates but might not affect pregnancy rates. Fertil Steril 84: 130-140. doi: 10.1016/ j.fertnstert.2004.08.042

8. Karagiannidis A, Varsakeli $S$, Alexopoulos C, Amarantidis I. 2000. Seasonal variation in semen characteristics of Chios and Friesian rams in Greece. Small Rumin Res 37: 125-130. doi: 10.1016/S0921-4488(99)00143-1

9. Lue YH, Hikim AP, Swerdloff RS, Im $P$, Taing KS, Bui T, Leung A, Wang C. 1999. Single exposure to heat induces stage-specific germ cell apoptosis in rats: role of intratesticular testosterone on stage specificity. Endocrinology 140: 1709-1717. doi: 10.1210/endo.140.4.6629

10. MacLaren AP. 1988. Ram fertility in south-west Scotland. Brit Vet J 144: 4554. doi: 10.1016/0007-1935(88)90151-0

11. McGowan M, Galloway D, Taylor E, Entwistle K, Johnston P. 1995. The veterinary examination of bulls. Indooroopilly, Australia: Australian Association of Cattle Veterinarian. $84 \mathrm{p}$.

12. Paul C, Murray AA, Spears N, Saunders PT. 2008. A single, mild, transient scrotal heat stress causes DNA damage, subfertility and impairs formation of blastocysts in mice. Reproduction 136: 73-84. doi: 10.1530/ REP-08-0036

13. Rockett JC, Mapp FL, Garges JB, Luft JC, Mori C, Dix DJ. 2001. Effects of hyperthermia on spermatogenesis, apoptosis, gene expression, and fertility in adult male mice. Biol Reprod 65: 229239.

14. Sanford ML, Baker JS. 1990. Enhanced testosterone secretion in adult rams after establishment of a highfrequency, low-amplitude pattern of LH pulses in the nonbreeding season occurs without changes in the number or binding affinity of testicular LH receptors. Acta Endocrinol 122: 55-61. doi: 10.1530/ acta.0.1220055

15. Setchell BP. 1987. Spermatogenesis and spermatozoa. In: Austin CR, Short RV (eds). Germ cells and fertilization. Reproduction in mammals. UK: Cambridge University Press. p 63-101.

16. Setchell BP. 1998. The parkes lecture. Heat and the testis. J Reprod Fert 114: 179-194. doi: 10.1530/jrf.0.1140179

17. Setchell BP, Maddocks S, Brooks DE. 1994. Anatomy, vasculature, innervation, and fluids of the male reproductive tract. Vol 1. $2^{\text {nd }}$ ed. In: Knobil E, Neill JD (eds). The physiology of the reproduction. New York: Raven Press. 1063-1047 p. 
18. Sorensen AM. 1991. Reproducción animal. Principios y prácticas. México: McGraw-Hill. 539 p.

19. Williamson $P$. 1974. The fine structure of ejaculated ram spermatozoa following scrotal heating. J Reprod Fert 40: 191195. doi: 10.1530/jrf.0.0400191

20. Yaeram J, Setchell BP, Maddocks S. 2006. Effect of heat stress on the fertility of male mice in vivo and in vitro. Reprod Fertil Dev 18: 647-653.

21. Zhu B, Walker SK, Oakey H, Setchell BP, Maddocks S. 2004. Effect of paternal heat stress on the development in vitro of preimplantation embryos in the mouse. Andrologia 36: 384-394. doi: 10.1111/j.1439-0272.2004.00635.x 